\title{
Indices ecodopplercardiográficos de função diastólica de gatos saudáveis não sedados
}

\author{
Diastolic echodopplercardiographic indices of non-sedated healthy cats
}

\author{
Elaine Cristina Soares ${ }^{1}$ Maria Helena Matiko Akao Larsson ${ }^{2}$ Alexandre Gonçalves Teixeira Daniel ${ }^{3}$ \\ Mariana Moraes Fantazzini ${ }^{4}$ Fernanda Lie Yamaki ${ }^{1}$ Roberto Carvalho e Pereira ${ }^{1}$
}

\section{RESUMO}

\begin{abstract}
O propósito deste estudo foi determinar os parâmetros de velocidades de ondas $E$ e A, relação E/A, tempo de desaceleração da onda E e TRIV em gatos normais e não sedados, bem como correlacionar tais índices com a freqüência cardíaca. Estudaram-se 40 gatos hígidos, adultos, abrigados no gatil da Faculdade de Medicina Veterinária e Zootecnia da Universidade de São Paulo. O exame foi realizado com os animais contidos em decúbito lateral esquerdo. Para cada parâmetro, obtiveram-se média, desvio padrão, mediana, intervalo interquartílico e amplitude total. Observou-se boa correlação entre a frequência cardíaca e a velocidade da onda E, relação E/A e TRIV, ao passo que o tempo de desaceleração da onda $E$ e a velocidade da onda A não foram influenciados pela freqüência cardíaca. Os índices determinados permitirão caracterizar o padrão de anormalidade diastólica, freqüentemente associados às cardiomiopatias em felinos.
\end{abstract}

Palavras-chave: função diastólica, ecodopplercardiografia, gatos.

\section{ABSTRACT}

The purpose of this study was to determine values as $E$ and $A$ waves velocities, E/A ratio, $E$ wave deceleration time, and IVRT in healthy non-sedated cats, as well as correlate these indices to the heart rate. Forty healthy, adult cats housed in the cattery of the School of Veterinary Medicine of São Paulo University were studied. Mean, standard deviation, median, interquartilic interval and range were calculated. Doppler examination was performed with the cats restrained in left lateral recumbency. Good correlation between heart rate and E wave velocity, E/A ratio and IVRT was observed, whereas $E$ wave deceleration time and $A$ wave velocity were not affected by the heart rate. The estabilishment of these values will allow to define the pattern of the diastolic abnormality, usually related to cardiomyopathies in cats.

Key words: diastolic function, echodopplercardiography, cats.

\section{INTRODUÇÃO}

Conceitualmente, a diástole é o período que vai do fechamento da valva aórtica à abertura da valva mitral e é dividida em 4 fases, sendo: período de relaxamento isovolumétrico; fase de enchimento rápido; fase de enchimento lento e contração atrial. A fase que vai do período de relaxamento isovolumétrico até o início do enchimento rápido compreende o relaxamento ventricular, que é o primeiro componente da diástole e se constitui num processo ativo. O segundo componente, passivo, é a complacência ventricular (NISHIMURA \& HOUSMANS, 1989).

A disfunção diastólica do ventrículo esquerdo é responsável por grande parte dos sintomas de doenças cardiovasculares. Aproximadamente um terço dos pacientes humanos com insuficiência cardíaca congestiva tem função sistólica normal, sendo

\footnotetext{
${ }^{1}$ Faculdade de Medicina Veterinária e Zootecnia da Universidade de São Paulo, SP, Brasil. Autor para Correspondência: Elaine Cristina Soares - Rua Quixadá, 244, Alto da Mooca, 03350-060, São Paulo, SP, Brasil. E-mail: ecsoares@usp.br ${ }^{2}$ Departamento de Clínica Médica da Faculdade de Medicina Veterinária e Zootecnia da Universidade de São Paulo, São Paulo, SP, Brasil.

${ }^{3}$ Faculdade de Medicina Veterinária e Zootecnia da Universidade de São Paulo, São Paulo, SP, Brasil.

${ }^{4}$ Autônomo, São Paulo, SP, Brasil.
} 
que os sintomas apresentados são atribuídos à função diastólica anormal (NISHIMURA et al., 1989). Em felinos, esta última é uma condição particularmente importante, pois as doenças mais freqüentes nesta espécie, ou seja, as cardiomiopatias hipertrófica e restritiva têm sinais clínicos baseados, em grande parte, nas alterações de relaxamento e complacência ventriculares. Assim, a cardiomiopatia hipertrófica é caracterizada por alteração no primeiro componente da diástole, enquanto que a restritiva é caracterizada por anormalidade de complacência ventricular (BOON, 1998).

Atualmente, a forma mais viável e factível de avaliação da função diastólica é a analise do fluxo transmitral por meio da ecodopplercardiografia. Utilizase o doppler pulsado, com o volume de amostra entre as pontas dos folhetos da valva mitral, para obtenção do traçado que possibilite a mensuração da velocidade de enchimento diastólico inicial do ventrículo esquerdo, representado pela onda E, e a velocidade de enchimento ventricular durante a contração atrial, demonstrada pela onda A. Ainda, pode-se obter valores referentes à relação entre a velocidade da onda $\mathrm{E}$ e a velocidade da onda $\mathrm{A}$ (relação $\mathrm{E} / \mathrm{A}$ ), à desaceleração e ao tempo de desaceleração da onda E. Posicionando-se o volume da amostra entre o folheto septal da valva mitral e a via de saída do ventrículo esquerdo, registram-se, simultâneamente, os fluxos transmitral e aórtico, o que permite a obtenção do tempo de relaxamento isovolumétrico (TRIV) (MORCERF, 1996; BOON, 1998). Variações desses índices para além do limite de normalidade apontam para a disfunção diastólica e, dependendo do padrão de anormalidade, pode-se inferir que o indivíduo apresente comprometimento do relaxamento ventricular, ou seja, da fase ativa da diástole, ou que o mesmo possua menor complacência/distensibilidade ventricular, ou da fase passiva da diástole.

No entanto, fatores como idade, pré-carga, pós-carga, função sistólica e freqüência cardíaca podem influenciar os índices diastólicos (HARRISON et al., 1991; COURTOIS et al., 1992; OH et al., 1997). A freqüência cardíaca pode interferir nas velocidades das onda E e A, embora tenha pouco efeito no tempo de desaceleração (NISHIMURA et al., 1989). Com o aumento da freqüência cardíaca, a velocidade da onda A pode aumentar relativamente a velocidade da onda E. O período de relaxamento isovolumétrico também é influenciado pela freqüência cardíaca, isto é, a duração aumenta com a duração do ciclo cardíaco (NISHIMURA et al., 1989; SANTILLI \& BUSSADORI, 1998). Portanto, a determinação da influência da freqüência cardíaca é primordial, podendo-se atribuir as mudanças que a mesma provoca nos fluxos transvalvares a uma falsa disfunção diastólica.

A despeito da importância de tais índices, a literatura é escassa em estudos que definam os padrões em gatos sadios e não sedados (SANTILLI \& BUSSADORI, 1998). Assim, o objetivo deste estudo foi determinar valores normais da velocidade pico de onda $\mathrm{E}$, velocidade pico da onda $\mathrm{A}$, relação $\mathrm{E} / \mathrm{A}$, tempo de desaceleração da onda E, bem como definir o tempo de relaxamento isovolumétrico para gatos domésticos, sem influência de qualquer tipo de sedativo ou anestésico. Além disso, procurou-se avaliar a influência da freqüência cardíaca em cada um desses índices.

\section{MATERIAL E MÉTODOS}

Foram submetidos à ecocardiografia, 40 gatos adultos sadios, machos ou fêmeas, abrigados no gatil da Faculdade de Medicina Veterinária e Zootecnia da Universidade de São Paulo. A higidez dos animais foi comprovada por meio de exame físico, incluindo auscultação cárdio-pulmonar, hemograma, testes de função renal e hepática, e eletrocardiograma.

Para a realização do exame ecodopplercardiográfico, utilizou-se aparelho da marca HITACHI, modelo EUB -515A, com transdutor setorial de $5 \mathrm{Mhz}$. Os exames foram registrados sob a forma de fotos, obtidas com o uso de impressora SONY, modelo UP890 CE.

Os animais foram posicionados em decúbito lateral esquerdo, ligeiramente inclinados, permitindo a localização do transdutor entre os espaços intercostais do hemitórax esquerdo, obtendo-se, assim, os cortes apicais de quatro e cinco câmaras (MINORS \& O’GRADY., 1998; BOON, 1998). A partir do corte apical quatro câmaras, posicionou-se o volume de amostra do doppler pulsado entre as pontas dos folhetos da valva mitral, durante a diástole, para o registro do fluxo transmitral, ou seja, onda E e onda A. Foram então mensuradas a velocidade máxima da onda E, velocidade máxima da onda A, relação E/A e tempo de desaceleração da onda E.

Com ligeira inclinação do transdutor, obteve-se o corte cinco câmaras e com o volume de amostra no interior do ventrículo esquerdo, entre o folheto septal da valva mitral e a via de saída do ventrículo esquerdo, foi possível o registro simultâneo dos fluxos transmitral e aórtico. O TRIV foi mensurado como sendo o período entre o final do fluxo aórtico e o início do fluxo mitral. Em cada animal, os diferentes índices foram medidos três vezes para o cálculo da média. Em alguns animais, cuja freqüência cardíaca se 
apresentou muito elevada, prejudicando a distinção entre as ondas $\mathrm{E}$ e A, procedeu-se estímulo vagal, por meio de pressão sobre o bulbo ocular ou plano nasal.

Foi realizada estatística descritiva para cada uma das variáveis, incluindo média, desvio-padrão, mediana, intervalo interquartílico e amplitude total. Utilizou-se o teste de normalidade de Andersen-Darling para verificar a distribuição da freqüência cardíaca, velocidade de onda $E$, velocidade de onda $A$, relação E/A, tempo de desaceleração de onda E e TRIV. A correlação de Pearson foi utilizada para verificar a influência da freqüência cardíaca sobre as variáveis com distribuição normal (velocidade E, velocidade A e TRIV) e a correlação de Spearman foi utilizada para aquelas cuja distribuição não apresentava curva normal (relação E/A e tempo de desaceleração da onda E) (DORIA FILHO, 1999; MINITAB, 2004). Foi realizado teste de hipóteses para avaliar se o valor de r obtido se devia apenas à variabilidade das amostras ou se realmente existia correlação, considerando-se significante quando $\mathrm{P}<0,05$.

\section{RESULTADOS}

Quarenta por cento (16) dos animais apresentaram freqüência cardíaca elevada, mesmo após manobra vagal. Nestes animais, houve fusão das ondas E e A, dificultando a avaliação do fluxo transmitral no traçado dos mesmos. Assim, foi possível a avaliação da velocidade da onda $\mathrm{A}$, velocidade da onda E, relação E/A e tempo de desaceleração da onda E em 24 gatos . O TRIV foi mensurado em todos os animais estudados. Os dados referentes à análise descritva de cada um destes índices apresentam-se dispostos na tabela 1.

A freqüência cardíaca apresentou correlação positiva média com a velocidade máxima da onda $\mathrm{E}(\mathrm{r}=0,57 ; \mathrm{P}=0,035 ; \mathrm{n}=24)$ e fraca com a relação $\mathrm{E} /$ $\mathrm{A}(\mathrm{r}=0,41 ; \mathrm{P}=0,0449 ; \mathrm{n}=24)$; por outro lado, a freqüência cardíaca apresentou correlação negativa, fraca, com o tempo de relaxamento isovolumétrico $(\mathrm{r}=-0,34$; $\mathrm{P}=0,0280 ; \mathrm{n}=40$ ).

Não se observou correlação entre o tempo de desaceleração da onda $\mathrm{E}$ e a freqüência cardíaca ( $\mathrm{r}=-0,30 ; \mathrm{P}=0,1475 ; \mathrm{n}=24)$, o mesmo ocorrendo com a velocidade da onda $\mathrm{A}(\mathrm{r}=0,06 ; \mathrm{P}=0,7791 ; \mathrm{n}=24)$.

\section{DISCUSSÃO}

O presente estudo fornece dados dos índices diastólicos de gatos hígidos e não sedados. A literatura referente ao assunto é escassa, sendo que na maioria dos estudos os animais eram sedados ou anestesiados. Apenas SANTILLI \& BUSSADORI (1998) estudaram animais não sedados.

Ao comparar os dados obtidos com aqueles de SCHOBER et al.(2003) (Tabela 2), que estudaram sete gatos sedados, observa-se que os valores de velocidade da onda E, tempo de desaceleração da onda E e TRIV de ambos trabalhos são parecidos. No entanto, a média da velocidade pico da onda A, encontrada no presente estudo, foi maior que a do citado autor; conseqüentemente, o valor da relação E/ A obtido foi menor. Da mesma forma, BRIGHT et al. (1999) (Tabela 2), estudando 12 gatos anestesiados, encontraram valor de velocidade da onda A menor e relação E/A maior do que obtido neste trabalho. Esta diferença pode ser justificada pelo fato de os animais daqueles dois estudos terem sido anestesiados, o que levou à diminuição da freqüência cardíaca.

Segundo HARRISON et al.(1991), a interrelação entre a velocidade pico da onda A e FC pode ser explicada pelo fato de que um aumento na freqüência cardíaca poderia levar à alteração de relaxamento, com mudança do enchimento ventricular predominante do início para o final da diástole. Contudo, não se observou diminuição na velocidade pico da onda $\mathrm{E}$, o que indicaria relaxamento anormal.

Tabela 1 - Índices de função diastólica, obtidos por meio de Doppler Pulsado, de gatos sadios, não sedados. São Paulo, 2005.

\begin{tabular}{|c|c|c|c|c|c|c|}
\hline Índices & Número de animais & Média & Desvio padrão & Mediana & $\mathrm{Q} 3-\mathrm{Q} 1$ & Amplitude total \\
\hline FC (bpm) & 40 & 199,95 & 27,09 & 198,50 & 35,50 & 134 \\
\hline Vel E (m/s) & 24 & 0,65 & 0,13 & 0,64 & 0,205 & 0,43 \\
\hline Vel A (m/s) & 24 & 0,45 & 0,09 & 0,46 & 0,135 & 0,35 \\
\hline $\mathrm{E} / \mathrm{A}$ & 24 & 1,47 & 0,39 & 1,42 & 0,4 & 1,54 \\
\hline $\mathrm{T}$ des $\mathrm{E}(\mathrm{ms})$ & 24 & 72,29 & 9,56 & 74,00 & 20 & 33,00 \\
\hline TRIV (ms) & 40 & 68,75 & 19,14 & 70,00 & 0,02 & \\
\hline
\end{tabular}

FC: freqüência cardíaca; Vel E; velocidade máxima da onda E; Vel A: velocidade máxima da onda A; E/A: relação entre as velocidades máximas da onda E e da onda A; Tdes E: tempo de desaceleração da onda E; TRIV: tempo de relaxamento isovolumétrico; Q3 - Q1: intervalo interquartílico 
Outra possibilidade seria a diminuição do tempo de enchimento diastólico, que ocorreria devido ao aumento da freqüência cardíaca e que poderia levar ao esvaziamento incompleto do átrio, previamente à contração atrial. Assim, neste momento haveria um maior volume, o que levaria ao aumento do fluxo transmitral na fase tardia da diástole. Ainda, este aumento de volume causaria uma melhor atividade de contração atrial, de forma similar ao mecanismo de Frank-Starling. Outra possibilidade residiria no fato de que em FC elevadas, o ventrículo esquerdo pode estar relativamente vazio e num ponto baixo na curva pressão-volume, no momento da contração atrial . Este ventrículo, não completamente preenchido, pode ser mais complacente e capaz de receber um volume maior quando da contração atrial. No presente estudo, porém, não foi observada correlação entre a FC e a velocidade pico da onda $A$.

YAMAMOTO et al. (1993) ressaltaram que a importância da freqüência cardíaca sobre a velocidade do enchimento diastólico tardio reside não só no encurtamento ou prolongamento do período de enchimento diastólico, mas nas mudanças associadas à hemodinâmica. Assim, contratilidade do átrio esquerdo, complacência do ventrículo esquerdo, intervalo P-R e ritmo cardíaco podem influenciar a velocidade da onda A.

Talvez um motivo para não se encontrar aumento da velocidade da onda A, associado ao incremento da freqüência cardíaca, seja o fato de que a variação da mesma não tenha sido suficiente para provocar as alterações hemodinâmicas responsáveis pelo aumento do fluxo transmitral tardio. Os trabalhos que acusam a variação concomitante entre a velocidade de pico da onda A e FC foram realizados com a mesma, controlada por marcapasso (HARRISON et al.,1991).

Quanto à velocidade de enchimento diastólico inicial (onda E), encontraram-se valores similares aos descritos por SCHOBER et al. (2003) (tabela 2). Ao analisar-se a influência da freqüência cardíaca na velocidade pico da onda E, constatou-se correlação positiva entre ambas.

YAMAMOTO et al.(1993), em estudo experimental, no qual utilizaram 14 cães para verificar os efeitos da freqüência cardíaca no enchimento diastólico do ventrículo esquerdo, avaliado tanto pelo fluxo transmitral quanto por variáveis hemodinâmicas obtidas de forma invasiva, não encontraram correlação entre a média geral da velocidade máxima da onda E e a FC. Porém, observaram incremento da velocidade da onda E em cinco casos e diminuição em nove. Os autores concluíram, então, que a variação da FC pode levar a mudanças na velocidade de enchimento diastólico inicial, por meio de combinação das variáveis hemodinâmicas (pressão de enchimento, relaxamento e complacência do ventrículo esquerdo). Assim, a alteração no período de enchimento não parece exercer efeitos diretos sobre a velocidade máxima da onda E.

Além disso, fatores que controlam o gradiente atrioventricular, como a pressão e complacência do átrio esquerdo, pressão e volume sistólico final do átrio esquerdo, contratilidade e relaxamento do ventrículo esquerdo, no que diz respeito ao recuo elástico e sucção ventricular têm influência importante na velocidade da onda E. Embora as variáveis hemodinâmicas não tenham sido avaliadas concomitantemente ao estudo Doppler no presente trabalho, pode-se supor que o aumento da velocidade da onda E esteja associado a estas variáveis, como observado por YAMAMOTO et al. (1993).

Não se observou influência da FC no tempo de desaceleração da onda E. Em gatos e seres humanos, o aumento da freqüência cardíaca não afeta o tempo de desaceleração da onda E (SANTILLI \& BUSSADORI, 1998; NISHIMURA et al., 1989). Porém, os dados são controversos, uma vez que

Tabela 2 - Índices de função diastólica, obtidos por meio de Doppler Pulsado, e gatos saudáveis, segundo diferentes autores.

\begin{tabular}{lccl}
\hline \multicolumn{1}{r}{ Índices } & $\begin{array}{c}\text { SANTILLI e BUSSADORI (n=20) } \\
\text { sedados) }\end{array}$ & $\begin{array}{c}\text { SCHÖo } \\
\text { (sedados) }\end{array}$ & $\begin{array}{c}\text { BRIGHT et al. (n=12) } \\
\text { (anestesiados) }\end{array}$ \\
\hline FC & - & $132 \pm 15$ & - \\
Vel E (m/s) & $0,67 \pm 0,13$ & $0,67 \pm 0,14$ & $0,7 \pm 0,04$ \\
Vel A (m/s) & $0,59 \pm 0,14$ & $0,33 \pm 0,11$ & $0,29 \pm 0,04$ \\
E/A & $1,19 \pm 0,30$ & $2,27 \pm 1$ & $2,49 \pm 0,28$ \\
T des E (ms) & $59,9 \pm 14,07$ & $66 \pm 13$ & $120 \pm 10$ \\
TRIV (ms) & $55,4 \pm 13,24$ & $71 \pm 17$ & $45,7 \pm 3,3$ \\
\hline
\end{tabular}

Os valores representam média \pm desvio padrão.

FC: freqüência cardíaca; Vel E: velocidade máxima da onda E; Vel A: velocidade máxima da onda A; E/A: relação entre as velocidades máximas da onda E e A; T des E: tempo de desaceleração da onda E; TRIV: tempo de relaxamento isovolumétrico. 
YAMAMOTO et al. (1993) e SCHOBER et al. (2003) descreveram diminuição no tempo de desaceleração de acordo com o aumento da freqüência cardíaca, em humanos e felinos, respectivamente. Novamente, a discrepância de observação pode ser devido à influência de variáveis hemodinâmicas.

Sabe-se que o tempo de desaceleração da onda $\mathrm{E}$ é determinado pela complacência do ventrículo esquerdo, bem como pela pressão intra-atrial elevada. Assim, em muitos indivíduos com alta pressão média do átrio esquerdo, o tempo de desaceleração é curto, e o inverso ocorre se a pressão do átrio esquerdo for baixa (NISHIMURA et al.,1997). Já a diminuição da complacência do ventrículo esquerdo leva ao aumento da pressão mínima do ventrículo esquerdo (FRAGATA \& AREIAS, 1996), e isto faz com que ocorra uma diminuição do tempo de desaceleração, ou seja , as pressões se equilibram mais rapidamente.

No presente estudo, observou-se aumento da relação E/A com o aumento da freqüência cardíaca. Isto ocorreu porque, enquanto a velocidade máxima da onda E aumentava de acordo com a freqüência cardíaca, o mesmo não acontecia com a velocidade máxima da onda A. Conseqüentemente, a relação E/A apresentou correlação positiva com a FC.

O TRIV obtido foi semelhante àquele referido por SCHOBER et al. (2003) (tabela 2), sendo que quanto maior a freqüência cardíaca, menor esse valor (NISHIMURA et al., 1989; MORCERF, 1996). Taquicardia e estimulação simpática podem exacerbar o relaxamento isovolumétrico, reduzindo o tempo de enchimento diastólico e acelerando o recuo elástico diastólico precoce do ventrículo esquerdo. Em contrapartida, SCHOBER et al. (2003) não observaram influência da freqüência cardíaca sobre o TRIV, muito embora os autores tenham colocado em questão o fato dos animais estudados terem sido anestesiados. Questionaram também o tamanho da amostra, constituída por apenas sete animais, o que pode ter levado à diminuição do poder dos testes estatísticos. O referido índice sofre também influência de condições de sobrecarga de volume, levando ao aumento do tempo de relaxamento isovolumétrico (FRAGATA \& AREIAS, 1996). Embora avaliando de maneira diversa, GOLDEN \& BRIGHT (1990) também demonstraram boa correlação entre pré carga e tempo de relaxamento parcial (t1/2), que é o tempo necessário para a pressão do ventrículo esquerdo reduzir pela metade. Em contrapartida, não encontraram associação entre esse índice e a freqüência cardíaca. Assim sendo, estas variações individuais devem ser consideradas quando da interpretação do TRIV.
No decorrer do estudo, observou-se o surgimento de alguns fatores, que podem ser considerados limitantes. O primeiro deles é a fusão das ondas E e A, observada em animais taquicárdicos, impedindo a interpretação do fluxo transmitral, o que ocorreu em 16 gatos estudados. Este alto número se deve principalmente ao fato de os animais não terem sido submetidos à anestesia ou sedação.

O segundo fator limitante é o desconhecimento da idade exata de cada animal avaliado. Estudos mostram que a faixa etária pode influenciar esses índices; assim, no homem, o envelhecimento leva a um prolongamento do TRIV e do tempo de desaceleração da onda E, redução na velocidade pico da onda $E$, aumento na velocidade pico da onda A e diminuição da relação E/A (BRYG et al., 1987; SANTILLI \& BUSSADORI, 1998). Estas mudanças estão relacionadas ao aumento da massa e da espessura da parede do ventrículo esquerdo, o que determina aumento da sua resistência, perda do recuo elástico e do efeito de sucção, com hipercontratilidade atrial. Além disso, a pressão arterial sistêmica aumenta com a idade e isto tem como conseqüência o retardo do relaxamento e o aumento do volume sistólico final, causando um prolongamento do período de relaxamento isovolumétrico e redução da velocidade pico da onda E. Entretanto, SANTILLI \& BUSSADORI (1998), estudando gatos normais, não anestesiados, não encontraram influência da idade na primeira fase da diástole, representada pela onda E.

O terceiro fator é a posição do volume da amostra, que também influencia a velocidade do fluxo transmitral. Assim, a maior velocidade é obtida nas pontas dos folhetos valvares mitrais, ocorrendo uma diminuição conforme o volume de amostra é levado mais próximo ao anel mitral (BRYG et al., 1987; MORCERF, 1996). Neste estudo, procurou-se registrar o fluxo a partir da amostra posicionada entre os folhetos, mas devido ao fato de os animais não estarem sedados, a manutenção desse posicionamento se tornou relativamente difícil. Para contornar esta situação, procurou-se medir os parâmetros em três repetições.

Finalmente, acredita-se que, para que se possa avaliar acuradamente o efeito da freqüência cardíaca no fluxo transmitral, a mesma deva ser controlada por marcapasso, como relatado por HARRISON et al. (1991).

\section{CONCLUSÕES}

O presente estudo contribuiu para a definição dos índices ecodopplercardiográficos de 
função diastólica em felinos domésticos, em condição semelhante à encontrada rotineiramente na clínica de pequenos animais, ou seja, sem sedação ou anestesia. Pôde-se observar interferência da frequiência cardíaca sobre a velocidade pico da onda E, relação E/A, e TRIV. Acredita-se que, para que as correlações entre FC e índices diastólicos sejam definidas com maior acuidade, estudos posteriores que incluam o controle da FC bem como a análise invasiva das variáveis hemodinâmicas sejam necessários.

\section{REFERÊNCIAS}

BOON, J.A. Manual of veterinary echocardiography. Baltimore: Williams \& Wilkins, 1998. 478p.

BRIGHT, J.M. et al. Pulsed doppler assesment of left ventricular diastolic function in normal and cardiomyopathic cats. J Am Anim Hosp Assoc, v.35, p.285-291, 1999.

BRYG, R.J. et al. Effect of aging on left ventricular diastolic filling in normal subjects. Am J Cardiol, v.59, p.971-974, 1987.

COURTOIS, M. et al. Factors related to end-systolic volume are important determinants of peak early diastolic transmitral flow velocity. Circulation, v.85, p.1132-1138, 1992.

DORIA FILHO, U. Introdução à bioestatistica. 2.ed. São Paulo: Negócio, 1999. 152p.

FRAGATA, J.; AREIAS, J.C. Effects of gradual volume loading on left ventricular diastolic function in dogs: implications for the optimisation of cardiac output. Volume loading and diastolic function. Heart, v.75, p.352-357, 1996.

GOLDEN, A.L.; BRIGHT, J.M. Use of relaxation half-time as an index of ventricular relaxation in clinically normal cats and cats with hypertrophic cardiomyopathy. Am J Vet Res, v.51, n.9, p.1352-1356, 1990.

HARRISON, M.R. et al. Effect of heart rate on left ventricular diastolic transmitral flow velocity patterns assessed by doppler echocardiography in normal subjects. Am J Cardiol, v.67, p.622-627, 1991.
MINITAB - The student editon of MINITAB statistical software adapted for education. 14 release: user's manual. New York: Addison-Wesley, 2004. 642p.

MINORS, S.; O'GRADY, M.R. Resting and dobutamine stress echocardiographic factors associated with the development of occult dilated cardiomyopathy in healthy Doberman Pinscher dogs. J Vet Int Med, v.12, p.369-380, 1998.

MORCERF, F.A. Ecocardiografia. 2.ed. Rio de Janeiro: Revinter, 1996. 671p.

NISHIMURA, R.A.; HOUSMANS, P.R. Assesment of diastolic function of the heart: baxkground and current applications of doppler echocardiography. Part I. Physiologic and Pathophysiologic Features. Mayo Clin Proc, v.64, p.7181, 1989.

NISHIMURA, R.A. et al. Assesment of diastolic function of the heart: background and current applications of Doppler echocardiography. Part II: Clinical studies. Mayo Clin Proc, v.64, p.181-204, 1989.

NISHIMURA, R.A.; TAJIK, A.J. Evaluation of diastolic filling of left ventricle in health and disease: doppler echocardiographi is tht clinician's Rosetta stone. J Am Coll Cardiol, v.30, p.8-18, 1997.

$\mathrm{OH}$, J.K. et al. The noninvasive assessment of left ventricular diastolic function with two-dimensional and Dopple echocardiography. J Am Soc Echocardiogr, v.10, p.246270, 1997.

SANTILLI, R.A.; BUSSADORI, C. Doppler echocardiographic study of left ventricular diastole in nonanaesthetized healthy cats. Veterinary Journal, v.156, p.203-215, 1998.

SCHOBER, K.E. et al. Comparison between invasive hemodynamic measurements and noinvasive assessment of left ventricular diastolic function by use of Doppler echocardiography in healthy anesthetized cats. Am J Vet Res, v.64, p.93-103, 2003.

YAMAMOTO, K. et al. Effects of heart rate on left ventricular filling dynamics: assessment from simultaneous recordings of pulsed Doppler transmitral flow velocity pattern and haemodynamic variables. Cardiov Res, v.27, p.935-941, 1993. 